\title{
Pulsation \& Long-Periods in Three Nearby M Supergiants
}

\author{
M. A. Smith, T. J. Teays, L. L. Taylor, R. Wasatonic \\ Computer Sciences Corp., 10000A Aerospace Rd., Lanham MD 20706
}

E. F. Guinan

Astronomy Dept, Villanova Univ., Villanova, PA 19085

and S. Baliunas

Center for Astrophysics, 60 Garden St., Cambridge, MA 02138

\section{Introduction}

We report on pulsational variability found in our decade-long study of UV and optical RV, light, K-line data sets for $\alpha$ Ori, $\alpha$ Sco A, and $\alpha$ Her A. As summarized by Smith et al. (1990), our radial velocities are derived from McMath observations of five metallic lines near $\mathrm{H} \alpha$ plus $\mathrm{H} \alpha$ itself. Optical light curves were obtained by small photometric telescopes, and K-line emissions from the Mt. Wilson K-line project. UV continua at $3000 \AA$ and $\mathrm{k} / \mathrm{h}$ emission indices were obtained with the IUE LWP camera.

\section{2. "Long periods"}

That $\alpha$ Ori has a "long period" (better called cycles) has been known for 80 years. Villanova photometry shows that during the last decade it has had a value of $\sim 4$ years. This modulation dominates the $\mathrm{H} \alpha \mathrm{RVs}$ and K-emission fluxes. The resulting correlation is that strong K-emission occurs when positive $\mathrm{H} \alpha \mathrm{RVs}$ and faint light do. $\alpha$ Sco shows a similar "long period" of $\sim 6$ yrs. between metallic, $\mathrm{H} \alpha$ RVs and K-fluxes. $\alpha$ Her shows a $\sim 4$ yr. variation between $\mathrm{H} \alpha$ and $\mathrm{K}$.

The general relation between $\mathrm{H} \alpha$ and $\mathrm{K}$ indices suggests an association with magnetic activity. These correlations might arise from solar-like supergranulations into which boundaries the downdrafts flow and magnetic fluxes intensify.

\section{Pulsations}

Dupree et al. (1987, 1990) discovered a 1.1-1.2 yr. period in $\alpha$ Ori from UV and optical indices. The phasing of our RV curve with their light curve confirms their interpretation of fundamental-mode radial pulsation. This period is most visible in the optical light and RV curves. A PDM diagram of our Villanova photometry for $\alpha$ Ori shows a wandering of this periodicity between 0.85 and 1.1 yrs. An examination of the long-period subtracted RVs for all three star shows that a best-fit sinusoid fits the data in the main but with important departures. The 
RV curves show am/fm modulations, evidence of period-doubling, and missing cycles followed by a resumption of the previous phasing.

$\alpha$ Her shows dramatic departures from a simple oscillation. Although the RV curve shows a primary 0.9 yr. period, the optical light curve shows a complex behaviour which demands continuous coverage during the cycle even to define a mean brightness level. After a brief lull, the optical light dims, brightens, and dims a second time. During strong cycles (1994) even a secondary maximum and tertiary minimum can appear. These features are similar to the "extra bumps" that can appear in the rising or falling branch of the light curves of Mira variables and are attributable to atmospheric heating by multiple shocks.

Where do the multiple shocks come from? Bowen's (1992) HD models provide a possible answer for strong fundamental pulsations. His simulations often develop a series of three unevenly spaced shocks which are produced by a 3:1 near-resonance of pulsation energy near the atmospheric acoustic cut-off of $\sim 90$ days. The shocks propel material outward such that it returns 90 days later - which is indeed the value of the bump spacings observed in $\alpha$ Her's optical light curve! These models predict that the "short periods" are less likely to develop for stars of increasing mass. We note that $\alpha$ Her falls in between $\alpha$ Ori and the Mira variables in the H-R Diagram and seems to show hybrid features.

\section{Pulsational shocks}

The $\mathrm{Mg}$ II $\mathrm{h} / \mathrm{k}$ lines are strong coolants in the shocks generated by strong pulsations in late stars. The phase lag between $k$-emission maximum and optical light maximum minimum can be used to estimate the distance the shocks transit before dissipating. The k-emission curves lag consistently $0.4 \pm 0.1$ cycles behind the RV curves $(0.0 @ \mathrm{RV}(\max ))$.

Although the UV continuum light curves are nearly sinusoidal for $\alpha$ Ori and $\alpha$ Sco, the waveform for $\alpha$ Her shows a square-wave maximum. The brightening first appears $\sim 50$ days before the optical light maximum, during the first optical minimum. It attains the same level and remains constant for the next for 4 months. The light maximum ends abruptly just as the k-emission flux is reaching its maximum, signalling a transition of optical thickness to thinness.

Our observations suggest that that the onset of a pulsation cycle in $\alpha \mathrm{Her}$ is first visible in the Balmer continuum when an optically thick shock emerges in the lower photosphere. At this time the product $\mathrm{T}_{\text {eff }}{ }^{4} \mathrm{R}^{2}$ is not yet at its maximum so the UV shock precedes the bright star phase. Likewise, the shock is still thin in the Paschen continuum, so optical observers still "look through" the shock (Luttermoser, priv. com.). When the shock finally dissipates and becomes optically thin in the near UV, $\mathrm{h} / \mathrm{k}$ emission grows to importance.

\section{References}

Bowen, G. 1992, in: Instabilities in Evolved Supergiants, ed. C. De Jager (Amsterdam: N. Holland), p. 104

Dupree, A., et al. 1990, ASP Conf. Series 11, p. 4

Smith, M. A., Patten, B. M., \& Goldberg, L. 1990, AJ, 98, 2233 